\title{
Privacy threat analysis of mobile social network data publishing
}

\begin{abstract}
With mobile phones becoming integral part of modern life, the popularity of mobile social networking has tremendously increased over the past few years, bringing with it many benefits but also new trepidations. In particular, privacy issues in mobile social networking has recently become a significant concern. In this paper we present our study on the privacy vulnerability of the mobile social network data publication with emphases on a re-identification and disclosure attacks. We present a new technique for uniquely identifying a targeted individual in the anonymized social network graph and empirically demonstrate the capability of the proposed approach using a very large social network datasets. The results show that the proposed approach can uniquely re-identify a target on anonymized social network data with high success rate.
\end{abstract}

Keyword: Mobile social network; Social network data publication; Privacy attack; Reidentification attacks; Disclosure attacks 